\title{
RADIATION DOSE IN PAEDIATRIC COMPUTED TOMOGRAPHY: RISKS AND BENEFITS
}

\author{
G.I. Ogbole
}

Correspondence:

Dr. G.I. Ogbole

Department of Radiology

University College Hospital, Ibadan.

\begin{abstract}
Computed tomography (CT) is a powerful tool for the accurate and effective diagnosis and treatment of a variety of conditions because it allows high-resolution three-dimensional images to be acquired very quickly. However as the number of CT procedures performed globally have continued to increase; with growing concerns about patient protection. Currently, no system is in place to track patient doses and the lifetime cumulative dose from medical sources. The widespread use of CT even in developing countries has raised questions regarding the possible threat to public health especially in children. The best available risk estimates suggest that paediatric $\mathrm{CT}$ will result in significantly increased lifetime radiation risk over adult CT. Studies have shown that lower milliampere-second (mAs) settings can be used for children without significant loss of information. Although the risk-benefit balance is still strongly tilted toward benefit, there is still need for caution. Furthermore since the frequency of paediatric CT examinations is rapidly increasing, and estimates suggest that quantitative lifetime radiation risks for children are not negligible, efforts should be made toward more active reduction of CT exposure settings in paediatric patients. This article hopes to address this concerns and draw attention to the fact that children are not 'small adults' and should therefore be treated differently.
\end{abstract}

Keywords: Computed Tomography, Radiation Risk, Radiation Dose, Patient Dose Reduction, Children.

\section{INTRODUCTION}

'One of the first duties of the physician is to educate the masses not to take medicine'. William Osler (1849 - 1919)

In 1972, Computed tomography (CT), a technique that produces non-superimposed, cross-sectional images of the body, was introduced into clinical practice. Its introduction revolutionized diagnostic radiology, as it experienced rapid technological developments (fast acquisition and reconstruction times, spiral acquisition mode, multislice capability). Its use has also in the last decade grown considerably. As a result, the numbers of examinations have increased to the extent that CT has made a substantial impact on not only patient care, but also patient and population exposure from medical $x$-rays. This relatively high dose modality, represents about $5-10 \%$ of all $\mathrm{x}$-ray examinations, but contributes between $41 \%$ and $75 \%$ of the collective dose from diagnostic radiology in some countries. ${ }^{1-3}$

It is estimated that more than 62 million CT scans per year are currently obtained in the United States, including at least 4 million for children. ${ }^{2}$
By its nature, CT involves larger radiation doses than the more common conventional $\mathrm{X}$-ray imaging procedures.

This review will examine the main clinical applications of CT scanning particularly in children. It will focus on the associated radiation doses they receive and the consequent cancer risks. It will also describe the various dose descriptors of CT and measures that can be employed to reduce radiation doses in children and the general patient population.

\section{The Use of CT}

The use of CT has increased rapidly, both globally as well as in Nigeria with more centers acquiring CT scanners in the last 3-5 years. In the United States CT scans have risen from an estimated 3 million per year in 1980 to more than 62 million currently. ${ }^{2}$ This sharp increase has been driven largely by advances in CT technology that make it extremely user-friendly, for both the patient and the physician. 
Most importantly the largest increases in CT use have been in the category of pediatric diagnosis ${ }^{4,5}$ and this trend can only be expected to continue as investigative medicine remains standard practice for the next few years.

The growth of CT use in children has been driven primarily by the decrease in the time needed to perform a scan - now less than 1 second - largely eliminating the need for anesthesia or sedation to prevent the child from moving during image acquisition ${ }^{4}$. In developed countries like the United States of America, the major growth area in CT use for children has been presurgical diagnosis of appendicitis, for which CT appears to be both accurate and cost-effective; though arguably no more so than ultrasonography in most cases. ${ }^{6}$

Other more common areas of CT use in children include the diagnosis, monitoring, treatment for infectious or inflammatory disorders, abdominal masses, seizures and injury-related conditions. It is also performed to evaluate blood vessels serving the brain, face or neck, the spinal cord and the spinal column. It is especially useful in cases of head injury, where the examination can display or rule out serious complications such as intracranial haemorrhage and other forms of brain injury. Except for the chest $\mathrm{x}$ ray, $\mathrm{CT}$ is the most commonly used imaging procedure for evaluating the chest. Using multidetector CT, it is possible to obtain very detailed images of the heart and mediastinum in children, even newborn infants. CT is well-suited for visualizing diseases or injury of important organs in the abdomen including the liver, kidney and spleen and to detect abdominal tumors, birth defects or stones in the urinary tract. In the pelvis, CT scans can help demonstrate cysts or tumors of the ovary or abnormalities of the bladder and pelvic bones. An international IAEA study has shown that some countries are over-exposing children to radiation when performing computed tomography (CT) scans. These children are receiving adult-sized radiation doses. In addition, the study showed that pediatric CT scans occur more frequently in Africa than in Asia and Eastern Europe. The frequency has been attributed to the limited availability of alternative medical imaging techniques, such as MRI and ultrasound, which do not involve ionizing radiation, or because some CT scans are performed unnecessarily ${ }^{7}$

The records at the reputedly largest teaching hospital in Nigeria show that the proportion of CT studies that are currently performed in children range between $14 \%$ and $18 \%$. This CT facility at the time of writing also did not use a documented guideline and protocol for imaging children.

\section{RADIATION DOSE}

The amount of radiation energy deposited in a medium is called the radiation dose. Different x-ray modalities address radiation dose in different ways. For example, in chest radiography it is the entrance exposure (not the dose) that is the commonly quoted comparison entity. In mammography, the average glandular dose is the standard measure of dose. The distribution of radiation dose in $\mathrm{CT}$ is markedly different than in radiography, because of the unique way in which radiation dose is deposited. There are three aspects of radiation dose in CT that are unique in comparison to $\mathrm{x}$-ray projection imaging ${ }^{8}$.

First, because a single CT image is acquired in a highly collimated manner, the volume of tissue that is irradiated by the primary x-ray beam is substantially small compared with, for example the average chest radiograph.

Second, the volume of tissue irradiated, is exposed to the $\mathrm{x}$-ray beam from almost all angles during the rotational acquisition, and this more evenly distributes the radiation dose to the tissues in the beam. In radiography, the tissue irradiated by the entrance beam experiences exponentially more dose than the tissue near the exit surface of the patient.

Finally, CT acquisition requires a high Signal to Noise Ratio (SNR) to achieve high contrast resolution, and therefore the radiation dose to the slice volume is much higher because the techniques used ( $\mathrm{kV}$ and $\mathrm{mAs})$ are higher. As a rough comparison, a typical PA (posterioranterior) chest radiograph may be acquired with the use of $120 \mathrm{kV}$ and $5 \mathrm{mAs}$ whereas a thoracic CT image is typically acquired at $120 \mathrm{kV}$ and $200 \mathrm{mAs} .^{8}$

\section{Dose Measurement}

Scattered radiation in CT is considerable, and it can be higher than the radiation dose from the primary beam. Scattered radiation is not confined to the collimated beam profile as primary $\mathrm{x}$-rays are, and therefore the acquisition of a CT slice delivers a substantial dose from scatter to adjacent tissues, outside the primary beam. Furthermore, most CT protocols call for the acquisition of a series of near-contiguous CT slices over the tissue volume under examination. For example, a protocol in which ten $10-\mathrm{mm}$ CT slices is acquired in the abdomen. The tissue in slice 5 will receive both primary and scattered radiation from its acquisition, but it will also receive the scattered radiation dose from slices 4 and 6 , and to a lesser extent from slices 3 and 7 , and so on.

It can be shown that that the C'T dose index (CTDI) provides a good approximation to the multiple scan 
average dose (MSAD) which is the average dose, at a particular depth from the surface, resulting from a large series of CT slices. The CTDI measurement protocol seeks to measure the scattered radiation dose from adjacent CT slices in a practical manner.

The CTDI is defined as the integral of the dose profile along a line parallel to the axis of rotation for a single scan, divided by the nominal slice thickness ${ }^{8,9}$.

The CTDI is also defined by the U.S. Food and Drug Agency as the radiation dose to any point in the patient including the scattered radiation contribution from 7 CT slices in both directions, for a total of 14 slices.

It is important to keep in mind that radiation dose in $\mathrm{CT}$ is proportional to the $\mathrm{mAs}$ used per slice. At the same KV, doubling of the $\mathrm{mAs}$ doubles the dose, and halving the mAs halves the dose ${ }^{8,9}$.

\section{Quantitative Measures}

Various other measures apart from the CTDI are used to describe the radiation dose delivered during CT scanning, the most relevant being absorbed dose and the effective dose.

The absorbed dose is the energy absorbed per unit of mass and is measured in grays (Gy). One gray equals 1 joule of radiation energy absorbed per kilogram. The organ dose (or the distribution of dose in the organ) will largely determine the level of risk to that organ from the radiation.

The effective dose, expressed in sieverts $(\mathrm{Sv})$, is used for dose distributions that are nothomogeneous (which is always the case with CT); it is designed to be proportional to a generic estimate of the overall harm to the patient caused by the radiation exposure. The effective dose allows for a rough comparison between different CT scenarios but provides only an approximate estimate of the true risk. For risk estimation, the organ dose is the preferred quantity.

Effective dose is defined as the radiation dose that, if received by the entire body, provides the same radiation risk (i.e., of cancer) as does the higher dose received by the limited part of the body actually exposed (i.e., the scanned volume)

Organ doses can be calculated or measured in anthropomorphic phantoms. ${ }^{9}$ Historically, CT doses have generally been (and still are) measured for a single slice in standard cylindrical acrylic phantoms ${ }^{10}$, the resulting quantity, the $\mathrm{CT}$ dose index, although useful for quality control, is not directly related to the organ dose or risk. ${ }^{11}$

\section{Typical Organ Doses}

As mentioned earlier Organ doses from CT scanning are considerably larger than those from corresponding conventional radiography. For example, a conventional anterior-posterior abdominal $\mathrm{x}$-ray examination results in a dose to the stomach of approximately $0.25 \mathrm{mGy}$, which is at least 50 times smaller than the corresponding stomach dose from an abdominal CT scan. The number of scans in a given study is, of course, an important factor in determining the dose. Mettler et al. ${ }^{12}$ reported that in virtually all patients undergoing CT of the abdomen or pelvis, more than one scan was obtained on the same day.

The radiation doses to particular organs from any given CT study depend on a number of factors. The most important are the number of scans, the tube current and scanning time in milliamp-seconds ( $\mathrm{mAs}$ ), the size of the patient, the axial scan range, the scan pitch (the degree of overlap between adjacent CT slices), the tube voltage in the kilovolt peaks $(\mathrm{kVp})$, and the specific design of the scanner being used ${ }^{12,13}$.

Many of these factors are under the control of the radiologist or radiographer. Ideally, they should be tailored to the type of study being performed and to the size of the particular patient, a practice that is increasing but is by no means universal. ${ }^{14}$ It is always the case that the relative noise or graininess in CT images will increase as the radiation dose decreases, which means that there will always be a tradeoff between the need for low-noise images and the desirability of using low doses of radiation. ${ }^{15}$

It is important to note here that $\mathrm{mAs}$ is a major factor affecting contrast resolution as it directly influences the number of $\mathrm{x}$-ray photons used to produce the CT image, thereby affecting the SNR and contrast resolution. Doubling the $\mathrm{mAs}$, (hence the dose) increases the SNR by $\sqrt{2}$ or $41 \%$ and contrast resolution consequently improves. ${ }^{\text {? }}$

The effective dose from CT can be multiple times larger than traditional plain film examinations, depending on the examination type. Table 1 compares typical plain film and CT effective doses for a few sample examinations for illustrative purposes. The data are from the International Commission on Radiation Protection (ICRP). ${ }^{16}$

Typical effective radiation doses in adults range from about $2 \mathrm{mSv}(0.2 \mathrm{rad})$ for head CTs to about 8 to 10 $\mathrm{mSv}$ for CTs of the chest, abdomen, or pelvis. These latter doses are high compared to those of natural background radiation, which is about $3 \mathrm{mSv} /$ year ${ }^{17}$. Thus; it would take a person 3.3 years to get the same 
amount of background radiation that an abdominal CT delivers in less than a minute. Even more striking is the understanding that a chest CT gives an effective dose of radiation equivalent to about 400 posteroanterior chest films.

Table 2 compares the effective dose from typical diagnostic procedures to the number of chest x-rays for equivalent effective dose, as well as to the time period for equivalent effective dose from natural background radiation. It must be borne in mind that these are adult estimates, and figures are expected to be greater for children due to their uniqueness in terms of size and radiosensitivity.

\begin{tabular}{lll}
\hline Exam type & $\begin{array}{l}\text { CT } \\
\text { Effective dose } \\
(\mathrm{mSv})\end{array}$ & $\begin{array}{l}\text { Plain film } \\
\text { Effective dose } \\
(\mathrm{mSv})\end{array}$ \\
\hline Chest & 8.0 & 0.02 \\
Head & 2.0 & 0.07 \\
Abdomen & 10.0 & 1.0 \\
Pelvis & 10.0 & 0.7 \\
\hline
\end{tabular}

Table 1. Typical effective dose from plain film and CT. Data from ICRP ${ }^{16}$

\section{CT Radiation Risks in Children: Need for Concern}

Depending on the machine settings, the organ being studied typically receives a radiation dose in the range of 15 millisieverts ( $\mathrm{mSv}$ ) (in an adult) to $30 \mathrm{mSv}$ (in a neonate) for a single CT scan, with an average of two to three CT scans per study. At these doses, the most likely (though small) risk is for radiation-induced carcinogenesis. ${ }^{15}$
Most of the quantitative information available regarding the risks of radiation-induced cancer comes from studies of survivors of the atomic bombs dropped in Hiroshima and Nagasaki in 1945 2 These survivors were exposed to a fairly uniform dose of radiation throughout the body, unlike the radiation from CT which is highly non-unniform. However these data are used as the basis for predicting radiation-related risks in a population because a substantial sub cohort of about 25,000 survivors ${ }^{18}$ received radiation doses similar to those of a CT examination i.e. less than 50 $\mathrm{mSv}$. Nevertheless there is little evidence that the risks for a specific organ are substantially influenced by exposure of other organs to radiation. Of course, the data from survivors of the atomic bombs show a significant increase in the overall risk of cancer in the subgroup of atomic-bomb survivors who received mean low dose radiation of about $40 \mathrm{mSv}{ }^{19,20}$ which approximates the relevant organ dose from a typical CT study involving two or three scans .

Although most of the quantitative estimates of the radiation-induced cancer risk are derived from analyses of atomic-bomb survivors, there are other supporting studies, including a current large-scale study of 400,000 radiation workers in the nuclear industry, ${ }^{20,21}$ who were exposed to an average dose of approximately $20 \mathrm{mSv}$ (a typical organ dose from a single CT scan for an adult). A significant association was reported between the radiation dose and mortality from cancer in this cohort (with a significant increase in the risk of cancer among workers who received doses between 5 and $150 \mathrm{mSv}$ ); the risks were quantitatively consistent with those reported for atomic-bomb survivors ${ }^{19}$.

\begin{tabular}{lccl}
\hline Diagnostic Procedure & $\begin{array}{l}\text { Typical effective } \\
\text { Dose }(\mathbf{m S v})\end{array}$ & $\begin{array}{l}\text { Number of Chest } \\
\text { X-rays (PA film) } \\
\text { for equivalent } \\
\text { effective dose } \boldsymbol{~}\end{array}$ & $\begin{array}{l}\text { Time Period for } \\
\text { Equivalent } \\
\text { Effective Dose } \\
\text { from Natural } \\
\text { Background } \\
\text { Radiation }\end{array}$ \\
\hline Chest x-ray (PA film) & 0.02 & 1 & 2.4 days \\
Skull x-ray & 0.07 & 4 & 8.5 days \\
Lumbar spine & 1.3 & 65 & 158 days \\
Upper G.I. exam & 3.0 & 150 & 1.0 year \\
Barium enema & 7.0 & 350 & 2.3 years \\
CT head & 2.0 & 100 & 243 days \\
CT abdomen & 10.0 & 500 & 3.3 years \\
\hline
\end{tabular}

Table 2. Effective Dose Comparisons ${ }^{8}$.

1. Assuming average effective dose from chest $\mathrm{x}$-ray (PA) of $0.02 \mathrm{mSv}$

2. Assuming average effective dose from natural background radiation of $3 \mathrm{mSv}$ per year 
The situation is even clearer for children, who are at greater risk than adults from a given dose of radiation, both because they are inherently more radiosensitive and because they more likely to live longer during which a radiation-induced cancer could develop. The National Research Council Committee on the Biological Effects of Ionizing Radiation has also estimated that children younger than 10 years of age are several times more sensitive to radiation than are adults ${ }^{21}$.

From these epidemiologic studies there is direct evidence that the organ doses corresponding to a common CT study (two or three scans, resulting in a dose in the range of 30 to $90 \mathrm{mSv}$ ) result in an increased risk of cancer. The evidence is reasonably convincing for adults and very convincing for children ${ }^{20-22}$.

\section{Cancer Risks associated with CT Scans}

No large-scale epidemiologic studies of the cancer risks associated with CT scans have been reported, however a study is ongoing that may elucidate this ${ }^{23,24}$. Although the results of such studies will not be available for some years, it is possible to estimate the cancer risks associated with the radiation exposure from any given C'T $\operatorname{scan}^{25}$ by estimating the organ doses involved and applying organ-specific cancer incidence or mortality data that were derived from studies of atomic-bomb survivors. As discussed above, the organ doses for a typical CT study involving two or three scans are in the range in which there is direct evidence of a statistically significantincrease in the risk of cancer, and the corresponding CT-related risks can thus be directly assessed from epidemiologic data, without the need to extrapolate measured risks to lower doses. ${ }^{26}$

Although the individual risk estimates are small, the concern about the risks from CT is related to the rapid increase in its use - small individual risks applied to an increasingly large population may create a public healthissue some years in the future.

\section{CT Radiation Risks: Greater in Children?}

In general, the doses and risks to children from CT examinations are not well understood, and are more difficult to calculate from CT scanner parameters. In a 2002 editorial, Slovis ${ }^{26}$ noted that there is an increased sensitivity to radiation in children compared to adults of up to 10 times, with girls being more radiosensitive than boys. Also, the lifetime radiation risks are higher for children because they have more expected years of life after the radiation exposure than adults ${ }^{25}$. Shrimpton et al. ${ }^{27}$ found through a 1989 UK survey that approximately 4\% of CT examinations were performed on children who were under 15 years of age. In 1998, Coren et al. ${ }^{28}$ reported a 63\% increase in requests for pediatric CT between 1991 and 1995 at a particular British hospital.

In 2001, Brenner et al. ${ }^{25}$ assessed the lifetime cancer mortality risks attributable to pediatric CT radiation. They reported that the estimated lifetime cancer mortality risks attributable to the radiation exposure from a CT examination of a one year old are 1 in 550 for a single abdominal CT, and one in 1500 for a head CT. They claimed that the risks are an order of magnitude higher than for adults, although this is a small increase in cancer mortality over the natural background rate. Brenner et al. again reported that of the 600,000 abdominal and head CT examinations performed on American patients less than 15 years of age, approximately 500 might ultimately die from cancer attributable to CT radiation ${ }^{25}$.

CT no doubt remains a very important modality for diagnosing disorders in pediatric patients. However, several studies suggest the need for caution because of the greater risk in children. Frush et a ${ }^{29}$ also has drawn interesting corollaries between the widespread use of $\mathrm{x}$-rays in the early days of radiography, before the damaging effects of radiation were understood fully, and today. They explored the risks of low-level radiation and CT and advocated following the ALARA (As Low As Reasonably Achievable) principle. Their research suggested a statistically significant, increased risk of fatal cancer from low-dose radiation in the range of 50 to $100 \mathrm{mSv}$. They explained that "a single CT of the abdomen could provide a dose of $11 \mathrm{mSv}$. If there are 3 phases in this examination, the actual dose is $33 \mathrm{mSv}(3 \times 11 \mathrm{mSv})$. If this child is 1 of the $30 \%$ who have 3 or more examinations, the lifetime dose is at least $100 \mathrm{mSv}$, clearly in the range of doses associated with induction of fatal cancer" ${ }^{29}$. These facts are crucial to understanding the risks that could be associated with indiscriminate use of repeated CT examination in children.

\section{Radiation Dose and Image Quality}

In general, the individual risk from the radiation dose associated with CT is thought to be small compared with the benefits that accurate diagnosis and treatment can provide. Still, unnecessary exposure to radiation during medical procedures should be avoided. Unnecessary radiation may be delivered when CT scanner parameters are not appropriately adjusted for children

Recommendations for reducing radiation exposure have stressed optimization of CT settings based on patient weight or diameter and the anatomic region of interest ${ }^{30,31}$. Specific recommendations have 
included reduction of both the tube current and the number of examinations performed with contrast material, use of a relatively large pitch, and elimination of inappropriate referrals for CT.

A critical issue in the evaluation of the radiation dose used in pediatric CT examinations is the size of the patient, since radiation dose is related to patient size. Studies have shown that the weighted CT dose index increased in small-diameter phantoms when examined with identical tube energy and current settings. This is because there is less intervening material to absorb the radiation in smaller phantoms. Because ionizing radiation causes more biologic effects in children, it is important to adapt the CT technical parameters to minimize radiation dose.

Studies undertaken to determine the relationship of radiation dose and image quality confirm that image noise increases as tube voltage decreases. These studies also demonstrate that beam energy has a direct influence on radiation dose. When tube voltage is decreased, radiation dose decreases in phantoms of all sizes. Their results confirm that tube current has a direct influence on radiation dose as has been reported by similar studies. ${ }^{32-38}$

They showed that it is possible to use a tube voltage as low as $80 \mathrm{kVp}$ and maintain image contrast in phantoms and that the increase in image noise is more obvious in larger phantoms than in smaller phantoms. Of note is the demonstration that there is no appreciable difference in image noise in the infant-sized (8-cm) phantoms at the 80 - and $120-\mathrm{kVp}$ settings. This likely reflects the fact that $\mathrm{x}$-ray photons are able to better penetrate smaller phantoms because there is less attenuation caused by intervening material.

The differences in image quality may not be as robust in patients because of differences in the administration of contrast medium or in breathing-related artifacts, both of which can affect the visibility of structures was an argument that was raised.

The results of this study and similar ones indicate that radiation dose can be decreased and acceptable image quality can be achieved in pediatric contrast-enhanced CT examinations by reducing tube voltage and tube current. These findings are of clinical importance as they could be used to develop clinical protocols in the pediatric population.

Although reduction in radiation dose is an important exercise, maintaining high quality of a diagnostic imaging study is also essential to provide an accurate and definitive diagnosis. There is need to achieve a fine balance between image quality and radiation dose.

\section{CT Radiation Dose Reduction}

While the benefits of CT exceed the harmful effects of radiation exposure in patients, increasing radiation doses to the population have raised a compelling case for reduction of radiation exposure from CT. ${ }^{29}$

Many studies have been performed to determine the possibility of reducing CT radiation doses for specific clinical indications especially in pediatric populations ${ }^{1}$ Since Infants and children represent a group of patients with a high radiosensitivity, ${ }^{26}$ dose reductions is especially important because of this sensitivity particularly in girls who are even more radiosensitive. ${ }^{26}$

Also because of they are expected to live longer, there is more time for most radiation induced cancers to develop ${ }^{39}$ than say for a 50 or 60 year old patient undergoing a CT examination. Radiologists must be aware of these facts and practice the ALARA principle, when it comes to deciding CT protocols and parameters $^{39}$.

The availability of multislice CT scanners has resulted in a considerable increase in the number of CT procedures per patient and per scanner. A recent international survey revealed that the mean effective dose to patients has increased from $7.4 \mathrm{mSv}$ at single slice helical CT to $8.1 \mathrm{mSv}$ in a multi-slice CT system ${ }^{40}$. Even in a developing country like Nigeria, newer available CT machines are either double or multi-slice thus leading to increased scan times and resultant dose increase. However, current commercially available multislice CT scanners have automatic tube current modulation capability (which is believed to be the most important contribution of industry toward radiation dose optimization) while simultaneously maintaining constant image quality regardless of patient attenuation characteristics ${ }^{40}$. However, the use, appropriateness and awareness of these functionalities may not yet be fully appreciated by a large proportion of users in Nigeria at this time.

Radiation exposure to patients remains the major disadvantage of computed tomography (CT) despite its high diagnostic value. CT scans are now the major cause of radiation for children. In order to minimize the radiation children receive from CT, two strategies can be applied. The first is the judicious use of CT, performing only examinations with absolute indications, and limiting these to the area in question ${ }^{41}$. This involves a multidisciplinary approach with the referring physician consulting the radiologist for their 
opinion as to the usefulness of requested investigations particularly in the instance of follow-up and repeat investigations.

The second one is modifying CT scan technical parameters in accordance with the size of the child, trying to avoid multiple sequences or cuts during the same CT study (for example, minimizing dual phase studies (pre- and post-contrast) ${ }^{6}$. This is primarily the responsibility of the radiologist.

European guidelines on quality criteria for CT have been published by the European Commission, in which two dose descriptors, weighted computed tomography dose index (CTDI) and dose-length product (DLP), were proposed as reference dose levels. CTDI provides the radiation dose from one slice at particular exposure settings and DLP characterizes the exposure from a complete examination. DLP increases in direct proportion to the number of cuts of the examination The ALARA (As Low As Reasonably Achievable) Conference in 2001 concluded that the highest priority should be given to reducing the radiation dose while still maintaining acceptable (diagnostic) image quality. The conventional way of achieving this is by using low dose CT ${ }^{41}$

For children, the situation may also be critical if scanning parameters are not adapted to their smaller size and increased radiation risk: the risk-benefit ratio may then no longer favor CT, if the cumulative doses delivered in the course of management are not monitored to keep it as low as reasonably achievable. The application of CT for young patients, patients with favorable prognosis and for frequent follow-up examinations will increase the radiation risk to the individual and the population. ${ }^{42}$

The lack of understanding of the approximate quantity of radiation delivered during various radiologic investigations is a universal problem amongst physicians as they often view CT studies in the same light as other radiologic procedures, even though radiation doses are typically much higher with CT. In a survey of radiologists and emergency-room physicians, ${ }^{43}$ about $75 \%$ of the entire group significantly underestimated the radiation dose from a CT scan, and $53 \%$ of radiologists and $91 \%$ of emergency-room physicians did not believe that CT scans increased the lifetime risk of cancer. In view of this, radiation risk education should be routinely conducted for the medical community to keep them updated and ensure they practice within acceptable guidelines.

Three ways to reduce the overall radiation dose from CT in the population have been suggested. The first is to reduce the CT-related dose in individual patients. The automatic exposure-control option on the latest generation of scanners is helping to address this concern.

The second is to replace CT use, when practical, with other options, such as ultrasonography and magnetic resonance imaging (MRI). Although the cost of MRI is more, there are some imaging scenarios in which MRI can simply replace CT. Some physicians and a large group of pediatric radiologists have suggested that perhaps one third of CT studies could be replaced by alternative approaches or not performed at all ${ }^{44,45}$. The third and most effective way to reduce the population dose from CT is simply to decrease the number of CT studies that are prescribed. From an individual standpoint, when a CT scan is justified by medical need, the associated risk is small relative to the diagnostic information obtained. However, if it is true that about one third of all CT scans are not justified by medical need, and it appears to be likely, ${ }^{45}$ perhaps 20 million adults and, crucially, more than 1 million children per year in the United States ${ }^{2}$ (as well as similar proportions in other countries) are being irradiated unnecessarily. Comparable data is not available in the Nigerian context but it may be understandable to think that the percentage may be sizable.

The widespread use of CT represents probably the single mostimportant advance in diagnostic radiology. However, as compared with plain-film radiography, CT involves much higher doses of radiation, resulting in a marked increase in radiation exposure in the population.

The increase in CT use and in the C'T-derived radiation dose in the population is occurring just as our understanding of the carcinogenic potential of low doses of x-ray radiation has improved substantially, particularly for children. This improved confidence in our understanding of the lifetime cancer risks from low doses of ionizing radiation has come about largely because of the length of follow-up of the atomicbomb survivors - now more than 50 years - and because of the consistency of the risk estimates with those from other large-scale epidemiologic studies. These considerations suggest that the estimated risks associated with CT are not hypothetical - that is, they are not based on models or major extrapolations in dose. Rather, they are based directly on measured excess radiation-related cancer rates among adults and children who in the past were exposed to the same range of organ doses as those delivered during CT studies ${ }^{46,47}$. With this background, and despite the fact that most diagnostic CT scans are associated with very favorable ratios of benefit to risk, there is a strong need to limit 
the number of CT studies that are being performed. Several authorities have questioned the use of CT, or the use of multiple CT scans, in a variety of contexts, ${ }^{48,49}$ and particularly its use as a primary diagnostic tool for acute appendicitis in children. ${ }^{6}$ But beyond these clinical issues, a problem arises when CT scans are requested in the practice of defensive medicine, or when a CT scan, justified in itself, is repeated as the patient passes through the medical system, often simply because of a lack of communication. Although in our environment this situation is yet to be a problem, perhaps due to the sparse availability of the facility and the high cost of a CT scan, a burden which is borne solely by the patient. Nevertheless its availability and use has continued to increase in the management of many pediatric and adult disorders nationwide.

\section{CONCLUSION}

While CT remains a crucial tool for paediatric diagnosis, it is important to minimize the radiation dose to children. Radiologists must continually think about reducing exposure as low as reasonably achievable (ALARA), by using exposure settings customized for children. All physicians who request paediatric CT should continually assess its appropriateness on a caseby-case basis. When used prudently, CT is a valuable imaging modality for both children and adults.

\section{REFERENCES}

1. Pages J, Buls N, Osteaux M. CT doses in children: a multicentre study. The British Journal of Radiology. 2003;76: 803-811

2. Brenner DJ and Hall EJ. Computed Tomography- An Increasing Source of Radiation Exposure.2007; 357:2277-2284

3. Wiest PW, Locken JA, Heintz PH, Mettler FA Jr. CT scanning: a major source of radiation exposure. Semin Ultrasound CT MR 2002; 23:402-410.

4. White KS. Helical/spiral CT scanning: a pediatric radiology perspective. Pediatr Radiol 1996; 26:5-14.

5. Linton OW, Mettler FA Jr. National conference on dose reduction in CT, with an emphasis on pediatric patients. AJR Am J Roentgenol 2003;181:321-329.

6. Stephen AE, Segev DL, Ryan DP, et al. The diagnosis of acute appendicitis in a pediatric population: to CT or not to CT. J Pediatr Surg 2003; 38:367-371.

7. Louise Potterton: New Study Shows Global Variation in Dose Levels for Child CT Scans, IAEA Division of Public Information. http:// www.european-hospital.com/en/article/ 7145.html accessed on 29 July 2010
8. Huda W, Atherton JV. Energy imparted in computed tomography. Med. phys 1994; 22:1263-1269.

9. Bushberg JT, Seibert JA, Leidholdt Jr. EM, Boone JM. The essential physics of medical imaging. Lippincott Williams \& Wilkins.2002; $2^{\text {nd }}$ ed. p362-367.

10. Groves AM, Owen KE, Courtney HM, et al. 16Detector multislice CT: dosimetry estimation by TLD measurement compared with Monte Carlo simulation. Br J Radiol 2004;77:662-665.

11. Brenner DJ. It is time to retire the computed tomography dose index (CTDI) for CT quality assurance and dose optimization. Med Phys 2006; 33:1189-1191.

12. Mettler FA Jr, Wiest PW, Locken JA, Kelsey CA. CT scanning: patterns of use and dose. J Radiol Prot 2000;20:353-359.

13. McNitt-Gray MF, AAPM/RSNA physics tutorial for residents - topics in CT: radiation dose in CT. Radiographics 2002; 22:1541-1553.

14. Paterson A, Frush DP, Donnelly LF. Helical CT of the body: are settings adjusted for pediatric patients? AJR Am J Roentgenol 2001; 176:297301.

15. Martin CJ, Sutton DG, Sharp PF. Balancing patient dose and image quality. Appl Radiat Isot 1999; 50:1-19.

16. Managing patient dose in computed tomography. A report of the International Commission on Radiological Protection. Ann ICRP. 2000; 30:7-45.

17. National Council on Radiation Protection and Measurements. Exposure of the population in the United States and Canada from natural background radiation, NCRP Report No. 94. Bethesda (MD): The Council; 1987. p. 148.

18. Preston DL, Pierce DA, Shimizu Y, et al. Effect of recent changes in atomic bomb survivor dosimetry on cancer mortality risk estimates. Radiat Res 2004; 162: 377-389.

19. Preston DL, Shimizu Y, Pierce DA, Suyama A, Mabuchi K. Studies of mortality of atomic bomb survivors. Report 13: Solid cancer and noncancer disease mortality: 1950-1997. Radiat Res 2003; 160: 381-407.

20. Pierce DA, Preston DL. Radiation-related cancer risks at low doses among atomic bomb survivors. Radiat Res 2000; 154: 178-186.

21. National Research Council, Committee on the Biological Effects of Ionizing Radiations. Health effects of exposure to low levels of ionizing radiation (BEIR V) Washington, DC: National Academy Press, 1990.

22. Cardis E, Vrijheid M, Blettner M, et al. Risk of cancer after low doses of ionizing radiation: 
retrospective cohort study in 15 countries. BM] 2005; 331: 77.

23. Giles J. Study warns of 'avoidable' risks of CT scans. Nature 2004; 431: 391-391

24. Brenner D, Elliston C, Hall E, Berdon W. Estimated risks of radiation-induced fatal cancer from pediatric CT. AJR Am J Roentgenol 2001;176:289-296.

25. Brenner DJ, Elliston CD, Hall EJ, Berdon WE. Estimates of the cancer risks from pediatric CT radiation are not merely theoretical. Med Phys 2001;28:2387-2388.

26. Slovis TL. The ALARA concept in pediatric CT: myth or reality? Radiology. 2002;223:5-6.

27. Shrimpton P, Jones D, Hillier M, Wall B, Le Heron J, Faulkner K. Survey of CT practice in the UK. Part 2: dosimetric aspects.: Chilton, England: National Radiological Protection Board; 1991.

28. Coren ME, $\mathrm{Ng} \mathrm{V}$, Rubens M, Rosenthal M, Bush A. The value of ultrafast computed tomography in the investigation of pediatric chest disease. Pediatr Pulmonol. 1998 ;26:389-395.

29. Frush DP, Donnelly LF, Rosen NS. Computed tomography and radiation risks: what pediatric health care providers should know. Paediatrics. 2003; 112: 951-957.

30. Brenner DJ, Doll R, Goodhead DT, et al. Cancer risks attributable to low doses of ionizing radiation: assessing what we really know. Proc Natl Acad Sci U S A 2003; 100:13761-13766.

31. Siegel MJ, Schmidt B, Bradley D, Suess C, Hildebolt C. Radiation dose and image quality in paediatric CT: effect of technical factors and phantom size and shape. Radiology. 2004; 233:515-522.

32. Brody AS. Thoracic CT technique in children. J Thorac Imaging 2001; 16:259- 268.

33. Chan CY, Wong YC, Chau LF, Yu SK, Lau PC. Radiation dose reduction in paediatric cranial CT. Pediatr Radiol 1999; 29:770-775.

34. Kamel IR, Hernandez RJ, Martin JE, Schlesinger AE, Niklason LT, Guire KE. Radiation dose reduction in CT of the pediatric pelvis. Radiology 1994; 190:683-687.

35. Lucaya J, Piqueras J, Garcia-Pena P, Enriquez G, Garcia-Macias M, Sotil J. Low-dose highresolution CT of the chest in children and young adults: dose, cooperation, artifact incidence, and image quality. AJR Am J Roentgenol 2000; 175:985-992.

36. Boone JM, Geraghty EM, Seibert JA, WoottonGorges SL. Dose reduction in pediatric CT: a rational approach. Radiology 2003; 228:352-360.
37. Nickoloff EL, Dutta AK, Zheng FL. Influence of phantom diameter, $\mathrm{kVp}$, and scan mode upon computed tomography dose index. Med Phys 2003; 30:395-402.

38. Huda W, Scalzetti EM, Levin G. Technique factors and image quality as functions of patient weight at abdominal CT. Radiology 2000; 217:430-435.

39. Katra MK, Maher MM, Toth TL. Hanberg LM, Blake MA, Shepard J, Saini S. Strategies for CT radiation dose optimization. Radiology 2004; 230:619-628.

40. Paterson A., Frush DP .Dose reduction in pediatric MDCT: general principles. Clin Radiol. 2007; 62:507-517

41. Karabulut N, Aryyurek M. Low dose CT: practices and strategies of radiologists in university hospitals. Diagn. Interv. Radiol 2006; 12:3-8

42. Jiménez S, Jiménez J R, Crespo M, Santamarta E, Bousoño C, Rodríguez J. Computed tomography in children with cystic fibrosis: a new way to reduce radiation dose. Archives of disease in childhood 2005:91:388-390.

43. Prokop M. Radiation dose in Ct: risks and challenges. Radiologe 48:229-242.

44. Lee CI, Haims AH, Monico EP, Brink JA, Forman HP. Diagnostic CT scans: assessment of patient, physician, and radiologist awareness of radiation dose and possible risks. Radiology 2004;231:393-398

45. Radiation risks and pediatric computed tomography (CT): a guide for health care providers. Rockville, MD: National Cancer Institute. http://www.nci.nih.gov/cancertopics/ causes/radiation-risks-pediatric-CT

46. Slovis TL, Berdon WE. Panel discussion. Pediatr Radiol 2002; 32:242-244.

47. Managing patient dose in computed tomography. A report of the International Commission on Radiological Protection. Ann ICRP. 2000; 30(4):745.

48. Donnelly LF. Reducing radiation dose associated with pediatric CT by decreasing unnecessary examinations. AJR Am J Roentgenol 2005; 184:655-657.

49. Ruess L, Sivit CJ, Eichelberger MR, Gotschall CS, Taylor GA. Blunt abdominal trauma in children: impact of CT on operative and nonoperative management. AJR Am J Roentgenol 1997; 169:1011-1014. 